\title{
Geistige Behinderungen: ein transdisziplinärer Ansatz
}

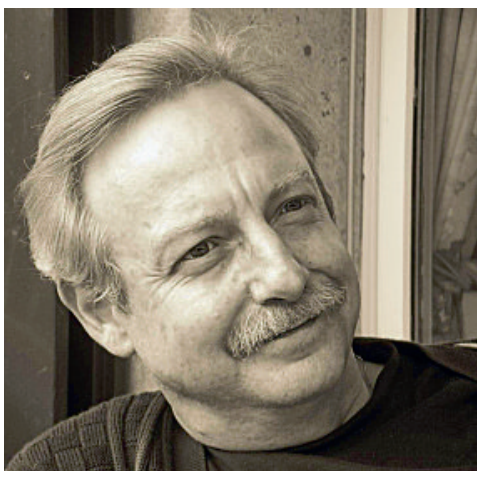

Claude-André Dessibourg
Vor mir sitzt Jeanne: Aus der Anamnese des hoch aufgeschossenen jungen Mädchens geht hervor, dass es an Neugeborenenkrämpfen litt und in Psychomotoriktherapie war. Es war einer Rekonstruktion des Kiefers aufgrund einer Mikrognathie unterzogen worden, und sein Zahnarzt hatte von einer auffällig geringen Zahnschmelzbildung gesprochen. Das Erlernen von Sprachen fällt ihm besonders schwer und es musste eine Klasse wiederholen und die Schule wechseln. Jeanne möchte Kleinkindererzieherin werden, doch ihre Eltern sind ehrgeizig und wünschen, dass sie die klassische Schulausbildung fortsetzt. Man steuert auf eine erneute Niederlage zu. Meine klinische Untersuchung ergibt den Befund eines diskreten motorischen Hemisphärensyndroms rechts. Die MRT zeigt eine ausgedehnte, frontotemporal ausgeprägte Lissenzephalie links, ohne irritatives Areal im EEG. Zweifelsfrei erklärt diese genetische Fehlbildung die Krampfanfälle, die Sprachstörungen, die psychomotorischen Störungen sowie die Dysmorphien. Die IV übernimmt nun die Kieferrekonstruktion, die zuvor als ästhetischer Eingriff beurteilt worden war, und das junge Mädchen erhält nach Gesprächen mit seinen Eltern eine Umschulung.

Michel ist 54 Jahre alt, mit Trisomie 21, und lebt in einem Heim. Seine Betreuerin berichtet, dass er sich zunehmend weniger für die Tätigkeiten in der Werkstatt interessiert, an Schlafstörungen leidet und sich mit kleinen Schritten bewegt. Klinisch zeigen sich neben den Stigmata des Down-Syndroms Rigor (Zahnradphänomen), ein Ruhetremor, archaische Reflexe usw. Es wird bei Michel ein Parkinson-Syndrom infolge der chronischen Einnahme von Neuroleptika diagnostiziert, das allerdings reversibel ist. Darüber hinaus sind die Anzeichen einer frühen Alzheimer-Krankheit festzustellen, wie es häufig bei Menschen mit Trisomie 21 auftritt; dies erfordert eine Anpassung der Aktivitäten und der Umgebung des Patienten.

Zwei Beispiele von Dutzenden in der täglichen Praxis eines Neurologen. Häufig sind die Probleme mehrschichtig und es bedarf einer dreifachen Diagnosestellung: somatisch, psychiatrisch und psychopädagogisch.

Derzeit besteht die grösste Gruppe der Menschen mit verminderter Intelligenz aus Erwachsenen. Es gibt kein medizinisches Fachgebiet, das sich speziell mit dieser Problematik befasst. Zwar werden diese Men- schen in der Kindheit gründlichen Untersuchungen unterzogen, eine spätere Aktualisierung ihres Status findet aber selten statt. Wie lautet die Diagnose Ihres Patienten? Sehr häufig haben Ergotherapeuten, Physiotherapeuten, Psychomotoriktherapeuten keinen Zugang zur Patientenakte. Sonderpädagogen werden mit der Verabreichung von Medikamenten (einschliesslich Notfallmedikamenten ...) und pflegerischen Leistungen beauftragt, obgleich sie keine entsprechende Ausbildung haben. Ein Antiepileptikum ist obsolet und dessen Blutwerte wurden niemals bestimmt, ein Antidepressivum wird seit fünf Jahren verabreicht, ohne dass wirklich klar ist, warum, ein anderer Patient nimmt wiederum offensichtlich zu hohe Dosen an Sedativa ein. Man sorgt für sofortige Abhilfe, stoppt einen epileptischen Anfall, erstickt eine Angst, verdoppelt und verdreifacht die symptomatischen Behandlungen. Ganz offensichtlich herrscht hinter dem Anschein einer perfekten Versorgung ein eklatanter Mangel an Zusammenarbeit zwischen Psychologen, Pädagogen und Ärzten deren Arbeit sich jedoch ergänzen sollte.

Anhand von 26 klinischen Fallbeispielen haben wir in unseren beiden jüngsten Büchern versucht, die Notwendigkeit eines transdisziplinären Ansatzes zu verdeutlichen. Während wir auf die Ergebnisse aus der Genetik und der Biotechnologie warten, müssen wir Verbindungen zwischen den Zünften errichten, über unseren engen Umkreis hinausschauen, die Einrichtung eines einheitlichen Patientendossiers unterstützen, Ausbildungen durchführen und in einen Dialog treten - ohne dabei je den ethischen Aspekt ausser Acht zu lassen. Wir sind alle, die Familien eingeschlossen, Kotherapeuten.

Aufgrund ihrer Komplexität bilden die geistigen Behinderungen die Schnittstelle zwischen unseren Disziplinen. Denn ohne Frage kann ein Einzelner nicht Experte auf den Gebieten der Neurowissenschaften, der Psychologie und der Erziehungswissenschaften zugleich sein. Interkulturalität ist hier aber mehr denn je sinnvoll und notwendig. Sich gegenseitig zu ignorieren, einerseits aus Gründen der ärztlichen Schweigepflicht, andererseits mit dem Argument des Dienstgeheimnisses, geht weit über schlichtes Zunftdenken hinaus: Es grenzt an einen Kunstfehler. Der Impuls entsteht vermutlich an der Synapse zwischen Empathie und den tektonischen
Claude-André Dessibourg*

\section{Platten des Wissens.}

Paris sein Buch «Handicap mental: approche transdisciplinaire» erschienen. 